\title{
Effect of Psychological Intervention on Emotion of Patients with Tumor Radiotherapy
}

\author{
Chao Wang*, Bo Xiang, Bo Han \\ Affiliated Qingdao Central Hospital, Qingdao University, Qingdao 266042, Shandong Province, China \\ *Corresponding author: Chao Wang, 1443787033@qq.com
}

\begin{abstract}
Objective: To explore the emotional impact of psychological nursing intervention on patients with malignant tumor radiotherapy, and further verify the specific application value of this nursing model in the oncology department. Methods: A total of 129 patients with tumor radiotherapy admitted to our hospital from June 2018 to June 2021 were selected as subjects, and they were divided into control group and observation group by random number method. Among them, 64 cases in the control group received routine clinical nursing. 65 cases in the observation group received psychological nursing intervention. Then, the clinical nursing effect, self-rating anxiety scale (SAS) score, self-rating depression scale (SDS) score, immune function level change condition and clinical nursing satisfaction were compared under the two nursing modes, in order to fully verify the clinical application value of psychological nursing intervention mode for patients with cancer radiotherapy. Results: Comparing the clinical indexes of the two groups, SDS and SAS scores of the observation group were significantly lower than those of the control group. In terms of nursing satisfaction, the observation group is $96.92 \%$ and the control group is $90.62 \%$, the difference was significant, and had clinical statistical significance $(P<0.05)$; After the comprehensive evaluation of the immune function of the two groups, it was found that the observation group was significantly better than the control group, and the difference between the two groups was statistically significant $(P<0.05)$. Conclusion: In the mode of psychological nursing, medical staff can carry out precise psychological nursing intervention and nutritional nursing for patients according to the specific conditions and treatment process of patients, which can greatly promote the psychological stability of patients, significantly reduce patients' negative emotions. In addition, the scientific implementation of psychological nursing intervention in the treatment of tumor radiotherapy patients can effectively strengthen the improvement of the immune function of patients, which has a very important application value to improve the physical function of patients, promotes the patient's body rehabilitation, and is worth in the field of widespread application.
\end{abstract}

Keywords: Psychological nursing; Chronic pelvic inflammatory disease; Radiotherapy

Publication date: September 2021; Online publication: September 30, 2021

\section{Introduction}

Tumor is one of the most common malignant diseases in the field of clinical medicine. The incidence of this disease is high and has a trend of gradual increase, which can seriously affect the physical and mental health of patients and cause a series of family and social problems. At present, in clinical treatment of tumor diseases, radiation therapy is often used to irradiation tumor lesions with radiation, inhibit or kill cancer cells, and then achieve the effect ${ }^{[1]}$. In the process of treating cancer patients with radiotherapy, it is also necessary to carry out psychological nursing intervention for patients, relieve the psychological pressure of patients, improve the compliance behavior of patients, so as to promote the rehabilitation of patients, which is reported as follows. 


\section{Materials and methods}

\subsection{General information}

129 tumor patients admitted to our hospital for radiotherapy from June 2018 to June 2021 were randomly divided into two groups. 64 cases in the control group, aged from 21 to 61 years, with an average age of (54 \pm 3.27 ) years, received routine clinical nursing; 65 cases in the observation group, aged from 26 to 64 years, with an average age of $(55 \pm 4.11)$ years, were treated with psychological nursing intervention. The research content has been approved by the ethics committee. All the recruited patients meet the clinical diagnostic criteria of tumor diseases and need radiotherapy treatment mode. There is no significant difference in the general data of patients $(\mathrm{P}>0.05)$.

\subsection{Methods}

The control group was given routine nursing.

Observation group added psychological nursing intervention on the basis of routine nursing, the methods are as follows:

\subsubsection{Establish a good relationship between nurses and patients}

Nurses should strengthen communication with patients, encourage patients to tell themselves their psychological feelings, and patiently listen to the inner voice of patients, so as to meet the actual psychological needs of patients to the greatest extent; At the same time, the causes of the negative emotions generated during the treatment of patients were analyzed, so as to provide targeted psychological counseling for patients; In addition, we should patiently answer the questions raised by patients, do not forget to pay attention to the explanation of radiotherapy knowledge, and formulate a detailed and careful treatment plan according to the patient's disease situation, so as to maximize the patient's confidence in healing. For patients who still have doubts, they can be guided to visit the radiotherapy center to understand the radiotherapy process from a macro perspective, so as to further improve the degree of treatment coordination.

\subsubsection{Correct treatment of adverse reactions in radiotherapy}

Before the implementation of radiotherapy, nursing staff should fully inform patients of the possible side effects and coping methods in the process of radiotherapy with what they have learned, so as to promote patients to treat the adverse reactions of radiotherapy correctly and reduce panic.

\subsubsection{Experience exchange}

Medical staff should encourage patients to actively participate in the cancer rehabilitation group of the hospital, and let the members of the group who have successfully received radiotherapy to transmit relevant experience, so as to effectively improve their degree of cooperation in radiotherapy treatment, improve their resistance, and regain their confidence in overcoming the disease.

\subsubsection{Psychological nursing}

The important role of psychological nursing is to relieve the patient's nervous mood, inform the patient of the treatment and treatment process. Different patients have different needs for psychological care. According to the specific situation and needs of patients, intervention nursing is carried out. During the treatment of patients, we should always pay attention to the patient's condition progress and psychological state, timely deal with and solve problems when we find them, so that patients can actively cooperate with the work of medical staff to strengthen the treatment effect. And after the patient was treated and discharged, help patients to overcome difficulties, regain the confidence to return to society, so as to achieve the purpose 
of psychological nursing intervention ${ }^{[2-3]}$.

\subsection{Observation indexes}

The scores of self-rating anxiety scale (SAS), self-rating depression scale (SDS), the level of immune function and clinical nursing satisfaction were observed in the two groups.

\subsection{Statistical methods}

SPSS18.0 statistical software was used to analyze and process the relevant data. The counting data was expressed in \%, the comparison was expressed by $\mathrm{X}^{2}$ test, and the measurement data was expressed by $\mathrm{t}$ test. The difference was statistically significant $(P<0.05) .(\bar{x} \pm s)$

\section{Results}

\subsection{Comparison of clinical nursing effect between the two groups}

The effective rate of clinical nursing under the two nursing modes was $98.46 \%$ in the observation group and $85.94 \%$ in the control group, and the $X^{2}$ test result was 41.4384 . The effectiveness of the observation group was significantly higher than that of the control group, and $P<0.001$. (Table 1.)

Table 1. Comparison of clinical nursing effect between two groups [N (\%)]

\begin{tabular}{cccccc}
\hline Group & Recovery & $\begin{array}{c}\text { Remarkable } \\
\text { effect }\end{array}$ & Effective & Inefficient & $\begin{array}{c}\text { Effective } \\
\text { rate }\end{array}$ \\
\hline Control group $(\mathrm{n}=64)$ & $30(46.88)$ & $12(18.75)$ & $13(20.31)$ & $9(14.06)$ & $55(85.94)$ \\
Observation group $(\mathrm{n}=64)$ & $35(53.85)$ & $17(26.15)$ & $12(18.46)$ & $1(1.54)$ & $64(98.46)$ \\
$\mathrm{X}^{2}$ & & & & & 41.4384 \\
$P$ & & & & & 0.0000 \\
\hline
\end{tabular}

\subsection{Comparison of SDS and SAS scores between the two groups}

Comparing the SDS score and SAS score of patients after the two nursing modes, the SDS score and SAS score of the observation group were significantly lower than those of the control group $(P<0.05)$. See Table 2.

Table 2. Comparison of SDS and SAS scores of the two groups ( $\bar{x} \pm s$, scores)

\begin{tabular}{|c|c|c|c|c|c|}
\hline \multirow{2}{*}{ Group } & \multirow{2}{*}{$\begin{array}{l}\text { Number } \\
\text { of cases }\end{array}$} & \multicolumn{2}{|c|}{ SDS score } & \multicolumn{2}{|c|}{ SAS score } \\
\hline & & Before nursing & After nursing & Before nursing & After nursing \\
\hline $\begin{array}{l}\text { Control } \\
\text { group }\end{array}$ & 64 & $65.1 \pm 6.5$ & $46.7 \pm 5.1$ & $62.5 \pm 6.5$ & $50.6 \pm 4.5$ \\
\hline $\begin{array}{l}\text { Observation } \\
\text { group }\end{array}$ & 65 & $64.9 \pm 6.2$ & $54.6 \pm 4.8$ & $61.8 \pm 7.0$ & $55.2 \pm 4.1$ \\
\hline $\mathrm{t}$ & & 0.1788 & 9.0610 & 0.5883 & 66.1694 \\
\hline$P$ & & 0.8583 & 0.0000 & 0.5574 & 0.0000 \\
\hline
\end{tabular}

\subsection{Comparison of nursing satisfaction between the two groups}

Compared with the control group, the nursing satisfaction of patients in the observation group was significantly higher than that in the control group $(96.92 \%>90.62 \%)$, the difference was statistically 
significant, $P<0.05$. See Table 3 .

Table 3. Survey and comparison of patients' nursing satisfaction [n (\%)]

\begin{tabular}{cccccc}
\hline Group & $\begin{array}{c}\text { Number of } \\
\text { cases }\end{array}$ & $\begin{array}{c}\text { Very } \\
\text { satisfied }\end{array}$ & Satisfied & Dissatisfied & Total satisfaction \\
\hline $\begin{array}{c}\text { Control } \\
\text { group }\end{array}$ & 64 & $36(56.25)$ & $22(34.37)$ & $6(9.37)$ & $58 / 90.62$ \\
$\begin{array}{c}\text { Observation } \\
\text { group }\end{array}$ & 65 & $43(66.15)$ & $20(30.76)$ & $2(3.07)$ & $63 / 96.92$ \\
$\mathrm{X}^{2}$ & & & & & 47.0870 \\
$P$ & & & & & $<0.001$ \\
\hline
\end{tabular}

\subsection{Comparison of immune function between the two groups}

After the evaluation of the immune function of the two groups of patients receiving different nursing modes, it was found that the immune function level of the observation group was significantly higher than that of the control group, and the difference between the two groups was statistically significant $(P<0.05)$, as shown in Table 4.

Table 4. Comparison of immune function of patients under two modes of care $\left({ }^{\bar{x}} \pm s\right)$

\begin{tabular}{lcccc}
\hline Group & Number of cases & CD3+ & CD4+ & CD8+ \\
\hline Control group & 64 & $45.41 \pm 6.74$ & $21.63 \pm 5.07$ & $23.19 \pm 6.15$ \\
Observation group & 65 & $82.87 \pm 9.83$ & $29.77 \pm 8.84$ & $26.88 \pm 8.88$ \\
$t$ & & 25.2051 & 6.4021 & 2.7397 \\
$P$ & & 0.0000 & 0.0000 & 0.0070 \\
\hline
\end{tabular}

\section{Discussion}

Malignant tumor has become one of the three major diseases threatening human life and health. In the treatment of such diseases, the most commonly used clinical method is radiotherapy. Due to the poor understanding of radiotherapy knowledge in the past, many patients showed resistance and low cooperation in the course of radiotherapy treatment, which seriously affected the treatment effect. Therefore, it is necessary to carry out psychological nursing intervention in radiotherapy treatment ${ }^{[4-5]}$.

This study took 129 patients with tumor radiotherapy as the object, 64 cases received routine clinical nursing, and the other 65 cases received routine nursing + psychological nursing intervention, that is, on the basis of routine nursing mode, targeted psychological counseling, i.e. comprehensive nursing management, was provided for their bad psychology, so as to eliminate the negative emotions of patients and regain self-confidence. After a period of care, compared the two groups of patients with clinical nursing effect, self-rating anxiety scale (SAS) score, self-rating depression scale (SDS) score, immune function level changes and clinical nursing satisfaction, we can find that the clinical nursing effect, immune function level and clinical nursing satisfaction of the observation group were significantly higher than that of the control group. The SAS and SDS scores of the observation group were significantly lower than those of the control group, indicating that the nursing model has a good application effect ${ }^{[6-8]}$.

In a word, the application of psychological nursing intervention in the treatment of tumor radiotherapy patients has very important clinical application value to improve the prognosis and cure effect of patients, which is worthy of wide promotion and application in the clinical field. 


\section{Disclosure statement}

The author declares no conflict of interest.

\section{References}

[1] Li JP, Zhao ZZ, 2021, The Effect of Psychological Intervention on Emotion in Patients with Cancer Radiotherapy. Journal of Psychology, (07): 5.

[2] Ma CY, 2020, The Effect of Psychological Nursing Intervention on the Psychological State of Patients with Malignant Tumor Radiotherapy. Guide of China Medicine. (01):30.

[3] Gu SY, 2017, Research on the Effect of Psychological Intervention on Emotion and Immune Function in Patients with Cancer Radiotherapy. World Latest Medicine Information, (10): 6.

[4] Guo JX, Zhou AH, Jiang ZL, 2018, Analysis of the Impact of Psychological Nursing Intervention on Negative Emotions of Patients with Malignant Tumor after Radiotherapy. Journal of Imaging Research and Medical Applications, 2(20): 221-222.

[5] Huang XQ, Zhou SZ, Lv DL, 2018, A Study on the Effect of Comprehensive Psychological Intervention Combined with Flupentixol and Melitracine on Negative Emotions in Patients with Tumor Chemoradiotherapy. Journal of Shenyang Medical College, 20(6): 526-529.

[6] Song YH, 2018, Effect of Psychological Nursing Intervention Measures on Psychological Status of Patients with Malignant Tumor after Radiotherapy. Journal of Mathematical Medicine, 31(1): $145-146$.

[7] Ma CY, 2020, The Effect of Psychological Nursing Intervention on the Psychological State of Patients with Malignant Tumor Radiotherapy. Guide of China Medicine, 18(3): 338-339.

[8] Meng PT, 2019, Effect Analysis of Psychological Nursing Intervention on Psychological Status of Patients with Malignant Tumor after Radiotherapy. Smart Healthcare, 5(17): 145-146. 